\title{
HSV-2-infected monocytes and keratinocytes show different inflammatory reaction in response to Fas receptor stimulation
}

\author{
MAEGORZATA KRZYŻOWSKA ${ }^{1,2}$, ANNA WINNICKA ${ }^{3}$,WANDA STANKIEWICZ ${ }^{4}$ \\ 1Department of Preclinical Sciences, Faculty of Veterinary Medicine, Warsaw University of Life Sciences - SGGW, Warsaw, Poland \\ ${ }^{2}$ Department of Regenerative Medicine, Military Institute of Hygiene and Epidemiology, Warsaw, Poland \\ ${ }^{3}$ Department of Pathology and Veterinary Diagnostics, Faculty of Veterinary Medicine, Warsaw University of Life Sciences - SGGW, \\ Warsaw, Poland \\ ${ }^{4}$ Department of Microwave Safety, Military Institute of Hygiene and Epidemiology, Warsaw, Poland
}

\begin{abstract}
In the present paper we assessed the role of Fas in the expression of pro-inflammatory cytokines and chemokines during HSV-2 infection using keratinocyte and monocyte cells in vitro. While uninfected keratinocyte and monocyte cultures responded with apoptosis induction upon Fas receptor stimulation with cytotoxic antibody, HSV-2-infected monocytes, but not keratinocytes, were susceptible to Fas-induced apoptosis. Both uninfected, Fas-stimulated and HSV-2-infected keratinocytes and only infected monocytes up-regulated the expression of pro-inflammatory chemokines - chemokine $(C-X$ - $C$ motif) ligand 1 (CXCL1) and CXCL10 and cytokines - interferon $\alpha(I F N-\alpha)$ and tumour necrosis factor $\alpha(T N F-\alpha)$. $H S V-2$ infection of monocytes and keratinocytes led to up-regulation of TNF- $\alpha, I F N-\alpha, C X C L 1$ and CXCL10 expression. Stimulation of Fas receptor reduced the expression of tested cytokines and chemokines in HSV-2-infected keratinocyte cultures, but not in monocytes. Furthermore, while apoptosis blocking down-regulated the expression of IFN- $\alpha, C X C L 1$ and CXCL1O in HSV-2-infected monocytes, the keratinocytes showed the opposite effect for IFN- $\alpha$, and CXCL10. We conclude that the Fas/FasL pathway participates in controlling the pro-inflammatory response during HSV-2 infection, albeit its effect depends on the type of infected cells.
\end{abstract}

Key words: HSV-2, apoptosis, Fas, keratinocyte, monocyte.

(Centr Eur J Immunol 2013; 38 (2): 196-202)

\section{Introduction}

The FS7-associated cell surface antigen (Fas, also named CD95, APO-1 or TNFRSF6) belongs to a subgroup of the tumour necrosis factor receptor (TNF-R) family that contains an intra-cellular 'death domain' and can trigger apoptosis. Its physiological ligand, FasL (CD95L), is a member of the corresponding TNF cytokine family. The Fas/FasL pathway has been shown to play critical roles in the immune system, in particular in the killing of pathogen-infected cells and the death of autoreactive lymphocytes [1].

Emerging evidence indicates that several members of the TNF-R family, including some that are classified as 'death receptors' (e.g. TNF-R1), and their corresponding ligands play (either exclusively or in addition to their pro-death activity) non-apoptotic functions, such as the induction of cellular activation, proliferation, differentiation or migration $[2,3]$. It has been shown that Fas/FasL death receptors activate inflammatory or proliferative signalling via the prototypic proinflammatory transcription factor $N F-\kappa B$ or the mitogen-activated protein kinase (MAPK) family of kinases [4]. Farley et al. (2008) demonstrated that FasL elicits a pro-inflammatory reaction in human keratinocyte cell line and reconstructed human epidermis by triggering the expression of stress-responsive transcription factors, inflammatory cytokines and chemokines [5].

Correspondence: Małgorzata Krzyżowska, Department of Regenerative Medicine, Military Institute of Hygiene and Epidemiology, Kozielska 4, 01-163 Warsaw, Poland, e-mail: krzyzowskam@yahoo.com 
Herpes simplex virus (HSV) causes a contagious infection that affects approximately $60 \%$ to $95 \%$ of adults worldwide. HSV-1 is associated mainly with infections of the mouth, pharynx, face, eye, and central nervous system (CNS), while HSV-2 is associated with infections of the anogenital region, although both serotypes may infect both areas. HSV-2 infection occurs via direct contact with infected lesions or body fluids and enters the body at mucosal tissues or through small lesions. Epithelial cells and keratinocytes are the primary target cells for HSV-2, but it also infects neuronal and immune cells [6-8]. HSV-2, but not HSV-1, was shown to inhibit cell surface expression of FasL in human leucocytes and K562 leukaemic cell line [9]. However, clearance of HSV-2 infection in a murine model requires Fas and perforin-dependent cytotoxic mechanisms, since mice lacking both perforin- and Fas-mediated cytolytic mechanisms were unable to completely clear the infection from the vaginal tissue [10]. In the previous study, HSV2-infected keratinocytes and epithelial cells upregulated Fas and FasL, but were resistant to Fas-induced apoptosis [11]. Furthermore, lack of Fas or FasL expression during genital herpes infection in mice led to an excessive inflammatory reaction in comparison to HSV-2-infected wild-type mice [11].

The aim of this study was to assess the role of Fas/FasL pathway in apoptosis induction and expression of proinflammatory cytokines and chemokines using an in vitro model of HSV-2 infection of keratinocyte and monocyte cell lines.

\section{Material and methods}

\section{Virus}

The HSV-2 strain 333 was grown and titrated in African green monkey kidney cells (GMK-AH1) and prepared by one cycle of freezing-thawing and subsequent removal of cellular debris by centrifugation [11].

\section{Cell lines and in vitro HSV-2 infection}

The mouse monocyte RAW 264.7 cells were from ATCC (TIB-71) and mouse keratinocyte cell line 291.03C were kindly provided by M. Kulesz-Martin (Department of Dermatology, Oregon Health and Science University, Portland, USA). RAW 264.7 cells were maintained in RPMI-1640 medium with $10 \%$ foetal bovine serum (FBS) and $1 \%$ antibiotic (Gibco by Life Sciences Technologies, Carlsbad, CA, USA). The 291.03C line, which is a 7,12 dimethylbenz[a] anthracene-initiated clone derived from non-transformed 291 cells, was cultured in $\alpha$-MEM (Gibco) supplemented with 5\% FBS (Gibco), $10 \mathrm{ng} / \mathrm{ml}$ epidermal growth factor (Sigma, St. Luis, MO, USA) and 1\% antibiotic (Gibco). The cell lines were infected with HSV-2 333 strain at MOI = 1-5, incubated for up to 18 hours and then harvested by trypsinisation or scaping. To induce apoptosis via the Fas-dependent pathway, cell cultures were incubated with an anti- mouse Fas cytotoxic antibody (Jo-1 clone) at $10 \mu \mathrm{g} / \mathrm{ml}$. For blocking apoptosis, zVAD-fmk - a pan-caspase inhibitor (Sigma), was used at $40 \mu \mathrm{M}$. All experiments were repeated 3 to 5 times.

\section{Apoptosis detection}

Apoptotic cells were detected using M30 CytoDEATH Fluorescein kit (Roche, Indianapolis, IN, USA); the M30 antibody cytoDEATH recognises a specific cleavage site within cytokeratin 18 that is not detectable in native cytokeratin 18 of normal cells. Prior to staining the samples were permeabilised with Cytofix/Cytoperm fixation/permeabilization kit (Becton-Dickinson, Franklin Lakes, NJ, USA), according to the manufacturer's protocol and once stained, analysed in FACS Calibur (BD Biosciences, USA).

\section{Antibodies and flow cytometric analysis}

Tumour necrosis factor $\alpha$, interferon $\alpha$ (IFN- $\alpha$ ), chemokine (C-X-C motif) ligand $1 \mathrm{~b}$ (CXCL1b) and CXCL10 were detected using Cytofix/Cytoperm fixation/permeabilization kit with Golgi plug (BD Biosciences) and the following antibodies: polyclonal goat anti-GRO $\alpha$ (P-20; CXCL1), polyclonal goat anti-IP-10 (C19; CXCL10) (Santa Cruz), monoclonal rat anti-IFN- $\alpha$ (F18; AbD Serotec), PE-conjugated monoclonal rat anti-TNF- $\alpha$ antibody (TN3-19.12; BD Biosciences). Following incubation with primary antibodies, appropriate PE-conjugated antigoat or anti-rat antibodies were used, where necessary (BD Biosciences). HSV-2 antigens were detected using rabbit FITC-conjugated rabbit polyclonal anti-HSV-1/2 antibody (Dako, Glostrup, Denmark). The stained cell suspensions were analysed in the FACS Calibur (BD Biosciences) for the percentage of positively stained cells.

\section{Real time PCR}

Total RNA was extracted from cultured cells using RNeasy Mini Kit (QIAGEN, Germany). First-strand complementary DNA (cDNA) was synthesized with oligo(dT)12-18 as primers, using the SuperScript First-Strand Synthesis System according to the manufacturer's protocol (Invitrogen, UK). Real-time PCR was performed in LightCycler 2.0 system (Roche) using $20 \mu$ volume capillaries and Taq ReadyMix ${ }^{\mathrm{TM}}$ for Quantitative PCR (Sigma). Average threshold cycle (CT) values from the triplicate PCR reactions for each particular gene were normalized against the average $\mathrm{CT}$ values for GADPH from the same cDNA sample and shown as $2^{\Delta \mathrm{CT}}$ were $\Delta \mathrm{C}_{\mathrm{T}}=\mathrm{CT}_{\mathrm{GADPH}^{-}}$ $\mathrm{C}_{\mathrm{T} \text { gene }}$. The following specific primers were used: GADPH, F, 5'- GGTGAAGGTCGGTGTGAACGGA -3', R, 5' - TGTTAGTGGGGTCTCGCTCCTG-3', CXCL1, F, 5'GCTGGGATTCACCTCAAGAA-3', R, 5'-TGGGGACACCTTTTAGCATC-3', CXCL10, F, 5'-CATGGTCCTGAGACAAAAGT-3', R , 5'-TGATGACACAAGTTCTTCCA-3', IFN- $\alpha$, R, 5' GAGGAACTGGCAAAAGGATGGTGA-3', F, 5'-TGTTGTTGCTGATGGCCTGATTGT-3', TNF- $\alpha$, 

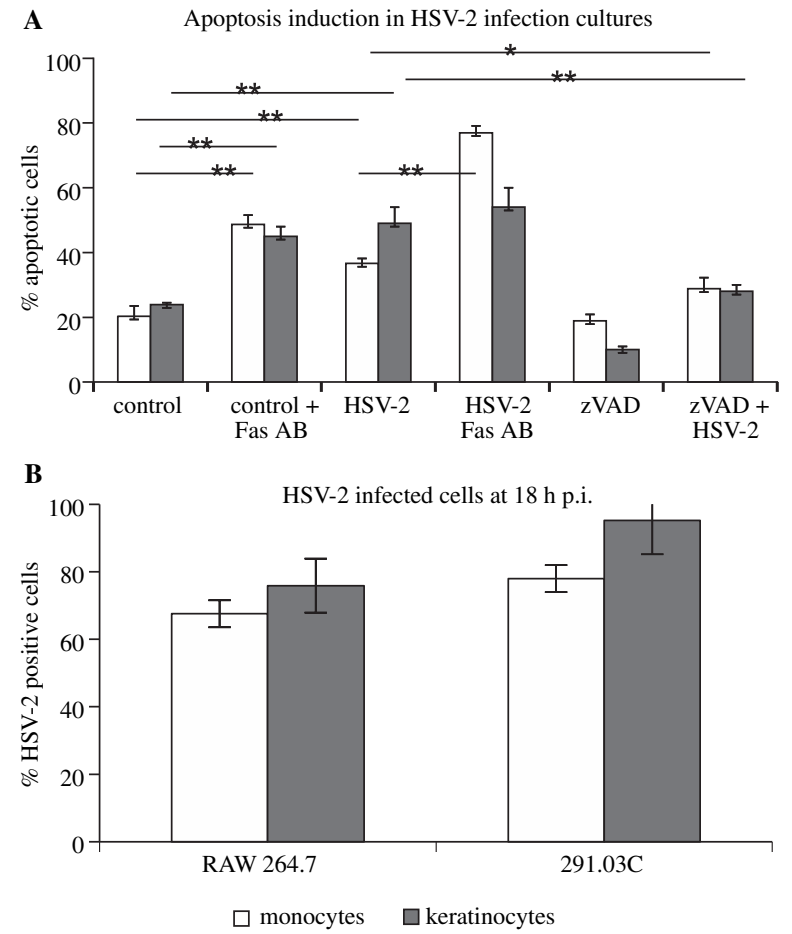

Fig. 1. A) Total percentage of M30-positive (apoptotic) cells in monocyte RAW 264.7 and keratinocyte $03 \mathrm{C}$ cell cultures at 18 hours after infection with HSV-2, subjected, or no, to ZVAD-fmk (ZVAD), a pan-inhibitor of caspases $(40 \mu \mathrm{M})$ or cytotoxic anti-Fas antibody $(10 \mu \mathrm{g} / \mathrm{ml})(\mathrm{Fas} \mathrm{Ab})$. Control uninfected cultures. B) Percentage of HSV-2-positive cells in monocytes and keratinocyte cultures at 18 hours after infection at M.O.I $=1$ and 5. Each bar represents the mean of 3 independent experiments $(N=3) \pm \mathrm{SEM}$. * represents significant differences with $p \leq 0.05$, while $* *$ means $p \leq 0.001$

\section{F, 5'-CCTTGTTGCCTCCTCTTTTGC-3', and R, 5'-TCAGT GTGATGTGATGTAGCGACAGCCTG-3' (Invitrogen).}

\section{Statistical methods}

All statistical analyses were conducted using the SPSS program (SPSS Inc., USA). Student's t-test and one-way ANOVA were used to determine significant $(p \leq 0.05)$ differences.

\section{Results}

We have previously shown that HSV-2 infected epithelial cells and keratinocytes up-regulate Fas and FasL but do not die through this receptor pathway [11]. Here, we have compared the apoptotic response of HSV-2-infected murine keratinocytes at 18 hours post infection (h p.i.) with HSV2 -infected murine monocytes in the presence of Fas cytotoxic antibody (Fig. 1A), expressed as the total percentage of M30-positive cells. Upon incubation with anti-mouse Fas cytotoxic antibody or infection with HSV-2, both monocyte and keratinocyte cultures show a significant increase in the total percentage of M30-positive (apoptotic cells) $(p<0.001)$ (Fig. 1A). However, addition of Fas cytotoxic antibody to HSV-2-infected monocytes and keratinocytes significantly increased apoptosis only for infected monocytes cultures $(p<0.001)$ (Fig. 1A). To further check whether HSV-2-infected keratinocytes and monocytes die through classical apoptotic pathways, we used a paninhibitor of caspases, z-VAD-fmk. Caspases are cysteine proteases responsible for the effector phase of apoptosis. Here, we showed that z-VAD-fmk significantly down-regulated the percentage of apoptotic keratinocytes and monocytes in the HSV-2-infected cultures $(p<0.05)$ (Fig. 1A). We further checked the percentage of HSV-2-infected monocytes and keratinocytes. At $24 \mathrm{~h}$ post infection, monocyte cultures infected at multiplicity of infection (M.O.I) = 1 showed approx. $60 \%$ of HSV-2-infected monocytes, and $75.89 \pm 8.1 \%$ at M.O.I $=5$ (Fig.1B). Keratinocytes infected with the same M.O.I showed $81.58 \pm 4.2 \%$ and 95.2 $\pm 9.1 \%$ of HSV-2-infected cells, respectively.

To evaluate the expression of chemokines and cytokines important for resolution of HSV-2 infection we employed real-time PCR technology and intracellular staining for cytokines and chemokines. Addition of anti-Fas cytotoxic antibody to control uninfected keratinocytes led to a significant up-regulation of TNF- $\alpha$, CXCL1 and CXCL10, but not IFN- $\alpha$ mRNA levels at 18 hours of incubation in comparison to uninfected, untreated cells ( $p \leq 0.05$ ) (Fig. 2). Similarly, upon Fas stimulation, we observed a significant up-regulation in the percentage of the cells expressing TNF- $\alpha$, CXCL1 and CXCL10, but not IFN- $\alpha(p \leq 0.05)$ (Fig. 2). On the contrary, Fas-antibody treated uninfected monocytes did not increase the expression of any tested cytokines or chemokines both at the mRNA levels and at the protein level (Fig. 3). HSV-2 infection of keratinocytes and monocytes in vitro led to a significant up-regulation of TNF- $\alpha$, IFN- $\alpha$, CXCL1 and CXCL10 mRNA expression levels at 18 hours of infection $(p \leq 0.001)$ and a significant up-regulation of cells expressing TNF- $\alpha$, IFN- $\alpha$, CXCL1 and CXCL10 ( $p \leq 0.05)$ (Figs. 2, 3). Addition of the cytotoxic anti-Fas antibody to HSV-2-infected keratinocyte cultures significantly abrogated TNF- $\alpha$, IFN- $\alpha$, CXCL1 and CXCL10 mRNA expression ( $p \leq 0.001$ ), followed by protein expression $(p \leq 0.05)$ (Fig. 2). Fas stimulation of HSV2-infected monocytes cultures resulted in significant downregulation of only IFN- $\alpha$ mRNA expression $(p \leq 0.001)$ (Fig. 3B).

To further check how blocking of apoptosis during HSV-2 infection influences production of pro-inflammatory cytokines and chemokines we compared mRNA levels of TNF- $\alpha$, IFN- $\alpha$, CXCL1 and CXCL10 in cultures subjected to a pan-caspase inhibitor, zVAD-fmk. Blocking of apoptosis in HSV-2-infected keratinocyte cultures led to 


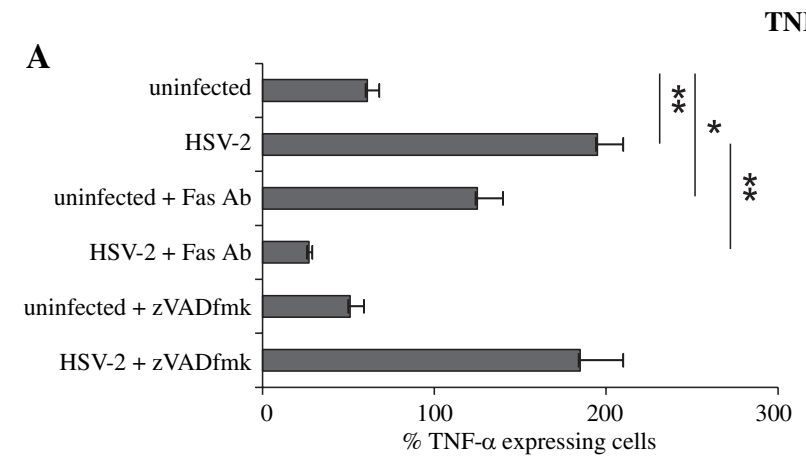

TNF- $\alpha$
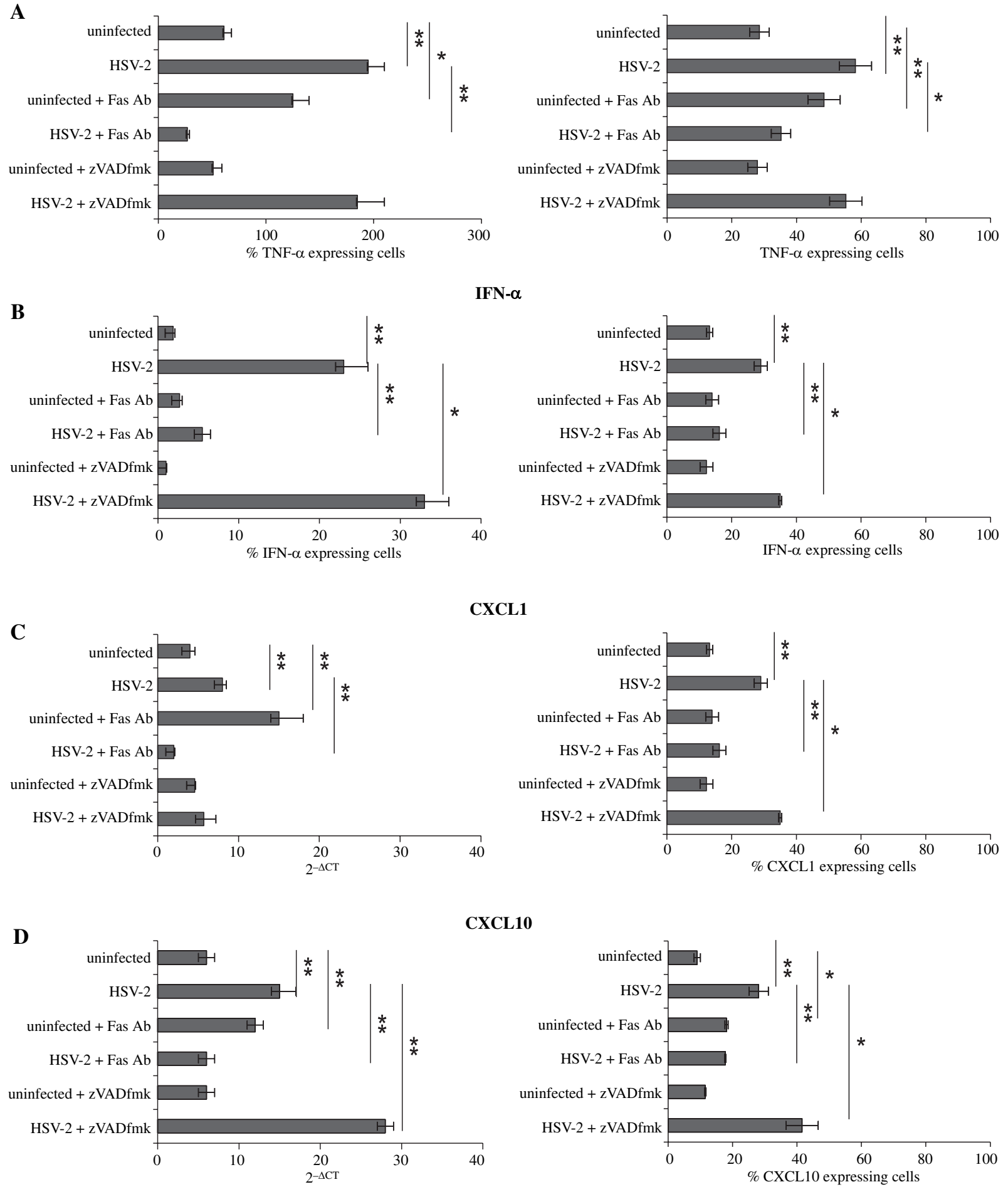

\section{CXCL10}

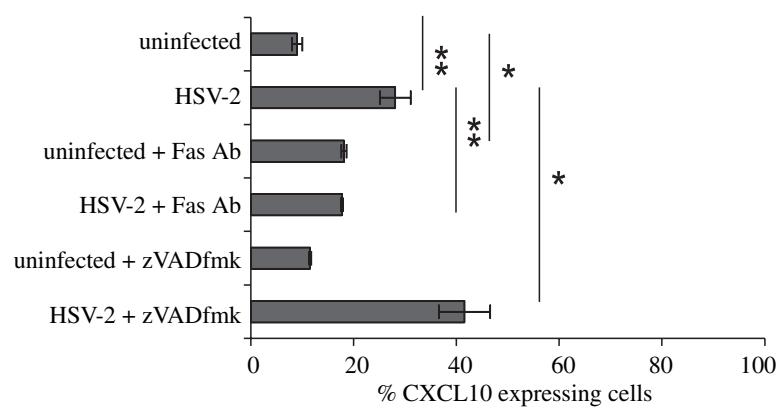

Fig. 2. Real-time PCR analysis (mRNA levels) and the percentage of cells positive for TNF- $\alpha$ (A) and IFN- $\alpha$ (B) CXCL1 (C), CXCL10 (D), in control and HSV-2-infected keratinocyte cell cultures at $18 \mathrm{~h}$ p.i. exposed or not, to anti-Fas cytotoxic antibody at $10 \mu \mathrm{g} / \mathrm{ml}$ or ZVAD-fmk at $40 \mu \mathrm{M}$. Each bar represents the mean $21^{-\Delta \mathrm{Ct}}$ from 3 separate experiments \pm SEM, in which RNA pooled from 2 experiments was used $(N=6) . *$ represents significant differences with $p \leq 0.05$, while $* *$ means $p \leq 0.001$ 


\section{TNF- $\alpha$}
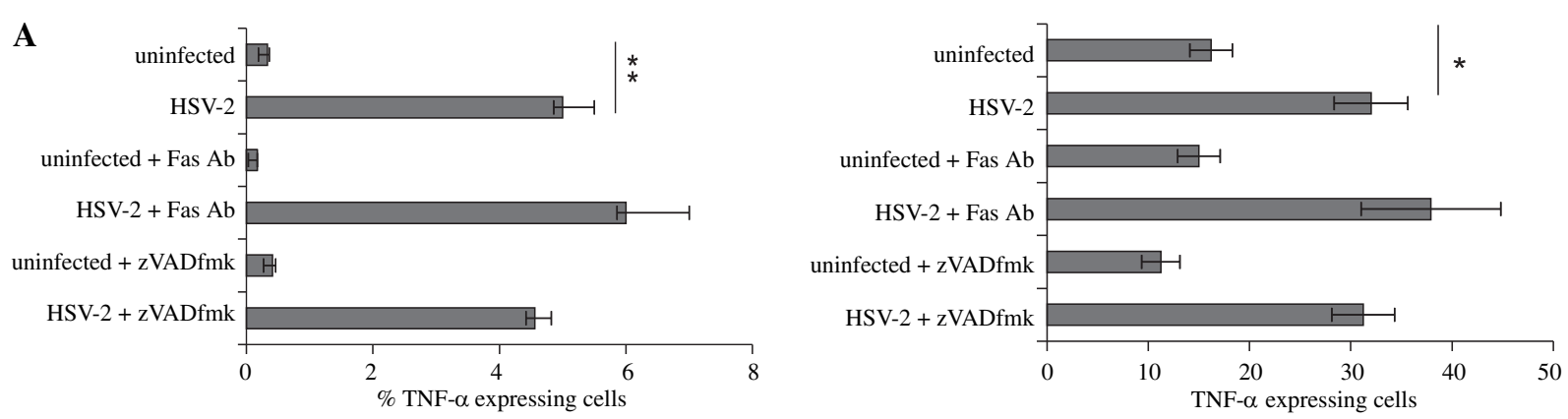

IFN- $\alpha$
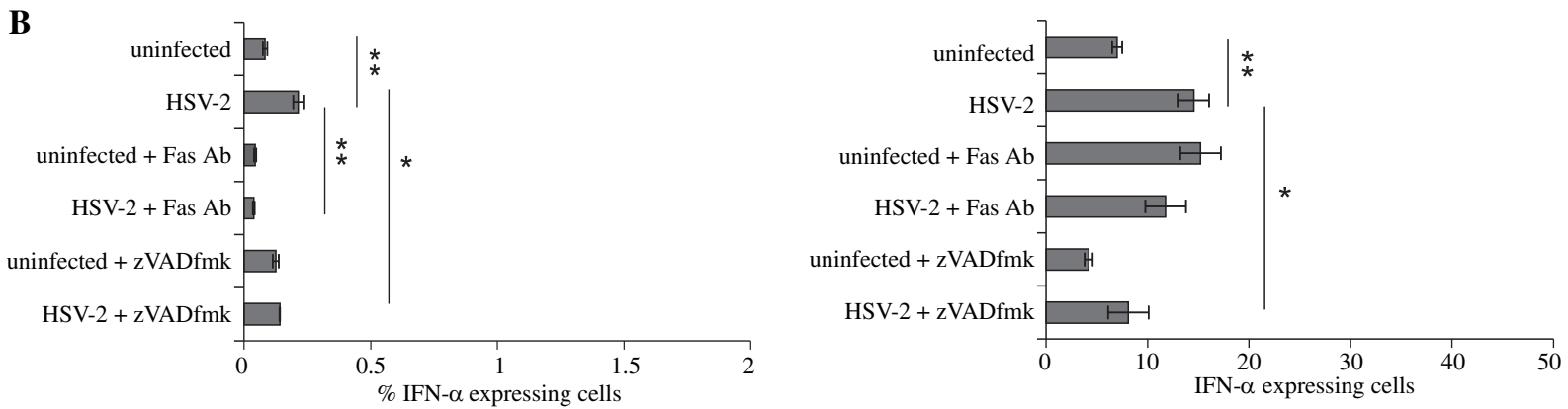

CXCL1
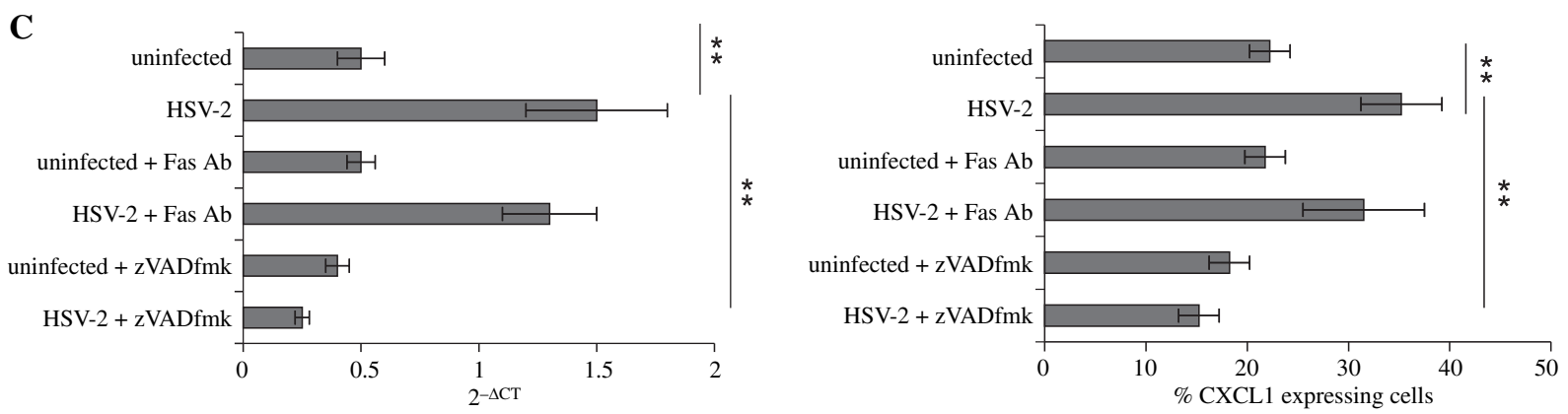

CXCL10

D


Fig. 3. Real-time PCR analysis (mRNA levels) and the percentage of cells positive for TNF- $\alpha$ (A) and IFN- $\alpha$ (B) CXCL1 (C), CXCL10 (D), mRNA levels in control and HSV-2-infected monocyte cell cultures at $18 \mathrm{~h}$ p.i. exposed or not, to anti-Fas cytotoxic antibody at $10 \mu \mathrm{g} / \mathrm{ml}$ or ZVAD-fmk at $40 \mu \mathrm{M}$. Each bar represents the mean $2^{-\Delta \mathrm{Ct}}$ from 3 separate experiments \pm SEM, in which RNA pooled from 2 experiments was used $(N=6)$.* represents significant differences with $p \leq 0.05$, while ** means $p \leq 0.001$ 
significant up-regulation of IFN- $\alpha$ and CXCL10 but not CXCL1 and TNF- $\alpha$, mRNA expression levels $(p \leq 0.05)$ (Fig. 2). The percentage of IFN- $\alpha$ and CXCL10 expressing cells also increased significantly at $18 \mathrm{~h}$ post infection $(p \leq 0.05)$ (Fig. 2). On the contrary, zVAD-fmk down-regulated the expression of IFN- $\alpha$, CXCL1 and CXCL10, but not TNF- $\alpha$ mRNA in HSV-2-infected monocytes $(p \leq 0.05)$ (Fig. 3). The patterns of mRNA expression were followed by the percentages of monocytes expressing IFN- $\alpha$, CXCL1 and CXCL10 ( $p \leq 0.05)$ (Fig. 3).

\section{Discussion}

The goal of this study was to investigate the role of Fas pathway in regulation of pro-inflammatory cytokines and chemokines expression during in vitro HSV-2 infection of mouse keratinocyte and monocytes. The cytokine and chemokine expression in the vagina during HSV-2 infection is necessary for mobilization and activation of innate immune cells including polymorphonuclear cells (PMNs), macrophages, and NK cells which play a vital role to limit viral replication and facilitate further adaptive immune response $[12,13]$.

Interferon type I is an important cytokine for HSV-2 immune response. Early production of IFN- $\alpha / \beta$ has been correlated with resistance to HSV infections [14]. HSV-2 infection of mice lacking the IFN- $\alpha / \beta$ receptor (IFN- $\alpha / \beta$ $\mathrm{R}-/-$ ) was characterized by enhanced genital viral replication and the disease progression. The mice were, however, able to mount a normal HSV-2-specific Th1 response [15]. TNF- $\alpha$ regulates leukocyte mobilization by inducing a number of factors, including cell adhesion molecules (ICAM-1 and VCAM-1), selectins (E and P selectins), and chemokine expression [16-18]. Tumour necrosis factor was also found to influence the macrophage activation during HSV-2 infection. In combination with IFN- $\alpha / \beta$, it synergistically enhanced the IFN-induced activation of macrophages [19].

CXCL1 expression is up-regulated in the vagina and CNS of mice during acute HSV-2 infection [18, 20]. Interferon inducible protein-10 (IP-10, CXCL10) is a chemokine that facilitates the recruitment of virus-specific T cells [20]. CXCL9 and CXCL10 are potent chemo-attractant molecules for NK and T cells during HSV-2 infection [21].

In our experimental model, consisting of murine keratinocytes and monocytes cell lines in vitro, we demonstrated an increased expression of CXCL1, CXCL10, TNF- $\alpha$ and IFN- $\alpha$ mRNAs in HSV-2-infected keratinocytes and monocytes, with keratinocytes showing higher inflammatory response than monocytes. This difference may result from the fact that monocytes support HSV-2 infection to a lesser degree [22]. Furthermore, the expression of CXCL1, CXCL10 and TNF- $\alpha$ increased significantly when uninfected keratinocytes were stimulated with the cytotoxic Fas antibody showing their ability to a pro-inflammatory response mediated via the Fas-dependent pathway. This effect was not observed for monocytes and confirms our previously obtained results, but also results by Farley et al. (2008) [5, 11].

Interestingly, the combination of HSV-2 infection and Fas pathway stimulation led to the down-regulation of a proinflammatory response in keratinocytes, while not in monocytes.

This was accompanied by the lack of apoptotic response to Fas stimulation in HSV-2-infected keratinocyte cultures. It was previously shown that HSV-2-infected epithelial HEp-2 cells show less apoptotic response due to activation of a known anti-apoptotic transcription factor in epithelia - NF-кB [23]. Also HSV-2-infected murine keratinocytes and epithelial cells in vitro upregulated Fas and FasL, but were resistant to Fas-induced apoptosis and exhibited an apoptosis prevention window correlating with up-regulated expression of Bcl-2, NF- $\mathrm{BB}$ and Akt activity [11]. Dufour et al. (2011) provided evidence that the ribonucleotide reductase R1 subunits of herpes simplex virus types 1 and 2 (HSV-1 and -2) protects cells against TNF- $\alpha$ and Fas ligand-induced apoptosis by interacting with caspase 8 [24]. However, blocking of apoptosis in HSV-2-infected keratinocyte cultures led to up-regulation of IFN- $\alpha$, CXCL1 and CXCL10 expression, in contrast to monocytes cultures, where blocking of apoptosis down-regulated expression of the same cytokines. Keratinocytes are main target cells for production of viral progeny. Therefore, it is necessary that no inflammatory response related with mobilization of early innate response is activated before the virus ends its replication cycle. This also indicates that other virus proteins can interfere with the keratinocyte inflammatory response in a manner allowing for maintaining a delicate balance between apoptosis induction and virus replication. Disturbing such a balance, for example by adding an external apoptosis blocking factor leads to inflammatory response. Furthermore, as shown before [11], HSV-2-infected keratinocytes up-regulate Fas and FasL, therefore the virus must block intrinsic mechanism of Fas-induced production of cytokines and chemokines as well as apoptosis. The opposite effect is observed for monocytes. During HSV infection macrophages are activated and possess an increased antiviral potential [19, 20]. Macrophages generally do not support HSV-2 replication [22]. Additionally, macrophages can inactivate virus replication by production of nitric oxide [20], but also by production of IFN- $\alpha / \beta$ and other pro-inflammatory cytokines and chemokines $[19,20]$. In our study, HSV-2-infected monocytes expressed mRNA of tested cytokines and chemokines, irrespectively of Fas stimulation but down-regulated the expression of IFN- $\alpha$, CXCL1 and CXCL10 when apoptosis was blocked extrinsically. This indicates that upon HSV-2 infection monocytes undergo apoptosis, followed by production of cytokines and chemokines, which are important for further activation of other components of innate and adaptive immune response. This apoptosis can be also induced by Fas-dependent path- 
way, as shown by the added apoptotic effect in the HSV-2infected monocytes cultures stimulated with cytotoxic antibody.

Our study shows that HSV-2 infection of different cell types leads to different inflammatory response of the infected cells. The Fas/FasL pathway may also participate in fine tuning of the local inflammatory response by regulating the inflammatory response of the HSV-2-infected cells, albeit it may also depend of the type of infected cell.

This work was supported by the National Science Centre grant no. N N401 178839.

The authors declare no conflict of interest.

\section{References}

1. Guicciardi ME, Gores GJ (2009): Life and death by death receptors. FASEB J 23: 1625-1637.

2. Aggarwal BB, Singh S, LaPushin R, et al. (1995): Fas antigen signals proliferation of normal human diploid fibroblast and its mechanism is different from tumor necrosis factor receptor. FEBS Letters 364: 5-8.

3. Peter ME, Budd RC, Desbarats J, et al. (2007): The CD95 receptor: apoptosis revisited. Cell 129: 447-450.

4. Wajant H, Pfizenmaier K, Scheurich P (2003): Non-apoptotic Fas signaling. Cytokine Growth Factor Rev 14: 53-66.

5. Farley SM, Dotson AD, Purdy DE, et al. (2006): Fas ligand elicits a caspase-independent proinflammatory response in human keratinocytes: implications for dermatitis. J Invest Dermatol 126: 2438-2451.

6. Gupta R, Warren T, Wald A (2007): Genital herpes. Lancet 370: $2127-2137$.

7. Crespi C, Cumberland W, Wald A, et al. (2007): Longitudinal study of herpes simplex virus type 2 infection using viral dynamic model ling. Sex Transm Infec 83: 359-364.

8 Larek-Rąpała A, Żaba R, Kowalczyk MJ, et al. (2011): Herpes simplex virus infection as a possible modulator of autoimmune diseases facilitated by human endogenous retroviruses. Post Dermatol Alergol 28: 313-316.

9. Sieg S, Yildirim Z, Smith D, et al. (1996): Herpes simplex virus type 2 inhibition of Fas ligand expression. J Virol 70: $8747-8751$.

10. Dobbs ME, Strasser JE, Chu CF, et al. (2005): Clearance of herpes simplex virus type 2 by CD8+ T cells requires gamma interferon and either perforin- or Fas-mediated cytolytic mechanisms. J Virol 79: 14546-14554.

11. Krzyzowska M, Shestakov A, Eriksson K, et al. (2011): Role of Fas/FasL in regulation of inflammation in vaginal tissue during HSV-2 infection. Cell Death Dis 2: 1-12

12. Parr MB, Parr EL (2003): Vaginal immunity in the HSV-2 mouse model. Int Rev Immunol 22: 43-63.

13. MasCasullo V, Fam E, Keller MJ, et al. (2005): Role of mucosal immunity preventing genital herpes infection. Viral Immunol 18: 595-606.

14. Ellermann-Eriksen S (2005): Macrophages and cytokines in the early defence against herpes simplex virus. Virology J 2: 59-79.

15. Svensson A, Bellner L, Magnusson M, et al. (2007): Role of IFN-alpha/beta signaling in the prevention of genital herpes virus type 2 infection. J Reprod Immunol 74: 114-123.
16. Horie YR, Chevenak P, Wolf R, et al. (1997): Lymphocytes mediate TNF - induced endothelial cell adhesion expression. Studies on SCID and RAG-1 mutant mice. J Immunol 159: 5053-5062.

17. Hornung F, Scala G, Lenardo MJ (2000): TNF- $\alpha$-induced secretion of $\mathrm{C}-\mathrm{C}$ chemokines modulates $\mathrm{C}-\mathrm{C}$ chemokine receptor 5 expression on peripheral blood lymphocytes. J Immunol 164: 6180-6187.

18. Thapa M, Carr DJ (2008): Chemokines and Chemokine Receptors Critical to Host Resistance following Genital Herpes Simplex Virus Type 2 (HSV-2) Infection. Open Immunol J 1: 33-41.

19. Ellermann-Eriksen S (1993): Autocrine secretion of interferon-alpha/beta and tumour necrosis factor-alpha synergistically activates mouse macrophages after infection with herpes simplex virus type 2. J Gen Virol 74: 2191-2199.

20. Peng T, Zhu J, Klock A, et al. (2009): Evasion of the mucosal innate immune system by herpes simplex virus type 2 . J Virol 83: 12559-12568.

21. Thapa M, Welner RS, Pelayo R, et al. (2008): CXCL9 and CXCL10 expression are critical for control of genital herpes simplex virus type 2 infection through mobilization of HSVspecific CTL and NK cells to the Nervous system. J Immunol 180: 1098-1106.

22. Sciortino MT, Perri D, Medici MA, et al. (2006): Role of Bcl2 expression for productive herpes simplex virus 2 replication. Virology 356: 136-146.

23. Yedowitz JC, Blaho JA (2005): Herpes simplex virus 2 modulates apoptosis and stimulates NF-kappaB nuclear translocation during infection in human epithelial HEp-2 cells. Virology 342: 297-310.

24. Dufour F, Bertrand L, Pearson A, et al. (2011): The ribonucleotide reductase R1 subunits of herpes simplex virus 1 and 2 protect cells against poly(I- C)-induced apoptosis. J Virol 85: 8689-8701. 